\title{
Synthesis of Facial Images with Foundation Make-Up
}

\author{
Motonori Doi ${ }^{1}$, Rie Ohtsuki ${ }^{2}$, Rie Hikima ${ }^{2}$, \\ Osamu Tanno ${ }^{2}$, and Shoji Tominaga ${ }^{3}$ \\ 1 Osaka Electro-Communication University, Osaka, Japan \\ ${ }^{2}$ Kanebo COSMETICS INC., Japan \\ ${ }^{3}$ Chiba University, Chiba, Japan
}

\begin{abstract}
A method is described for synthesizing color images of whole human face with foundation make-up by using bare face image and the surface-spectral reflectance of the bare cheek. The synthesis of made-up facial images is based on the estimation of skin color with foundation make-up and the control of skin texture. First, the made-up skin color is estimated from the spectral reflectance of the bare cheek and the optical properties of the foundation. The spectral reflectance of made-up skin is calculated by the Kubelka-Munk theory. Second, smooth texture control is done by the intensity change of layers in the multi-resolution analysis with the Daubechies wavelet. Luster is enhanced and acnes are attenuated by the texture control. Experimental results show the accurate estimation of made-up skin color and the effective texture control. It is shown that the made-up face images are rendered with sufficient accuracy.
\end{abstract}

Keywords: Color image, Facial image synthesis, Make-up foundation, Kubelka-Munk theory, Multi-resolution analysis, Texture synthesis.

\section{Introduction}

The analysis/synthesis of human skin color and texture is one of the most interesting topics for many fields including computer graphics, medical imaging, and cosmetic development. Especially, the synthesis of make-up skin color and texture are needed to examine the effect of make-up to human skin. Foundation is one of cosmetics to conceal undesirable color on skin and gives basic color and luster to the skin. It is important to evaluate the change of skin condition by the application of the foundation on the skin.

In a previous paper [1, we proposed a method for estimating the skin color with foundation make-up. The main idea of the method was based on the fact that skin color with foundation make-up could be computed from the measured reflectance of the bare skin and the optical properties of the foundation by the Kubelka-Munk theory. The made-up skin color without texture was estimated for small areas of cheek. We also analyzed skin texture based on the multi-resolution analysis (MRA) using wavelet transform [2].

A. Trémeau, R. Schettini, and S. Tominaga (Eds.): CCIW 2009, LNCS 5646, pp. 188 197, 2009.

(C) Springer-Verlag Berlin Heidelberg 2009 
The present paper describes a method for synthesizing color images of whole human face with foundation make-up by using bare face image and the surfacespectral reflectance of the bare cheek. It should be noted that the best way to show the effect of foundation application is to display the whole face image with the estimated skin color on a display device. We aim at developing a facial image synthesis system for displaying the effect of make-up foundation on human faces. However, it is difficult to acquire the detailed spectral reflectance data for all surface points on a human face. Moreover, the skin texture is altered with the applied foundation. For instance, acnes can be inconspicuous with foundation, and strong luster can exist in made-up face image. Therefore, the synthesis of made-up face images is based on the estimation of skin color with foundation and the control of skin texture.

\section{Synthesis of Made-Up Facial Images}

Fig 1] shows the procedure for synthesizing made-up facial images. The input data for the facial image synthesis are a bare face image and the surface-spectral reflectance of the bare skin. The representative reflectance is measured from a point on the cheek. First, the made-up skin color is estimated from the spectral reflectance of the bare skin and the optical properties of the foundation. The facial image is divided into block sub-images. Then, the skin color area is detected and converted to the estimated made-up color. The color distribution of the made-up facial image is smaller than the bare facial image. Therefore, the reduction of the color distribution for the entire skin area is done. Next, texture of the image is controlled by changing the intensity of the texture components in different levels of the MRA. This texture control works for attenuating undesirable spots on skin such as acnes, and enhancing luster patterns. Then, the texture-controlled sub-images are unified into whole facial image. Finally, a synthesized made-up face image is created by combining the skin area from the

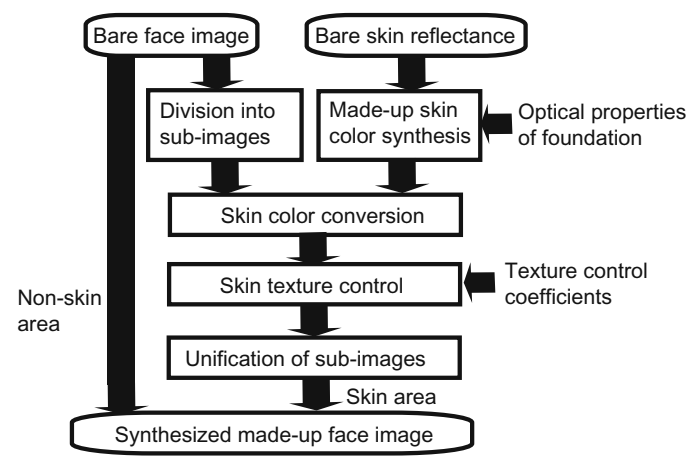

Fig. 1. Procedure for synthesizing made-up facial images 
unified image and the non-skin area, such as hair, eyes and background, from the original bare face image into whole human face image.

\subsection{Estimation of Made-Up Skin Color}

We estimate skin color based on the surface-spectral reflectance, because the spectral reflectance function provides the physical property inherent to skin surface. Therefore, we first estimate the surface-spectral reflectance with make-up foundation. The estimated reflectance is then converted to RGB values in considering illumination conditions.

In order to determine a relationship between the spectral reflectance function of skin with make-up and the optical properties of skin and foundation, an optics model is assumed for the skin with foundation make-up as shown in Fig.2, The skin optics model consists of two layers of foundation and skin, where the foundation layer is the outer layer contacting the air and the skin layer is located under the foundation layer. In this model, incident light is partly reflected at an interface between the foundation surface and the air. The light penetrating the interface is absorbed and scattered in the foundation layer. The light ray that reaches the skin surface is reflected and absorbed in skin layer.

The theory by Kubelka 344 is used for estimating the spectral reflectance of skin with foundation make-up based on the above optics model. In general, the optical values of reflectance and transmittance within a layer consisting of turbid materials can be calculated using the Kubelka-Munk theory, where we are not consider the complex path of scattered light inside the medium. We can derive the reflectance $R$ and the transmittance $T$ of the turbid layer with thickness $D$ from solving the above equations under some assumptions as follows

$$
\left.\begin{array}{l}
R=\frac{1}{a+b \operatorname{coth} b S D} \\
T=\frac{b}{a \sinh b S D+b \cosh b S D} \\
a=\frac{S+K}{S}, b=\sqrt{a^{2}-1}
\end{array}\right\}
$$

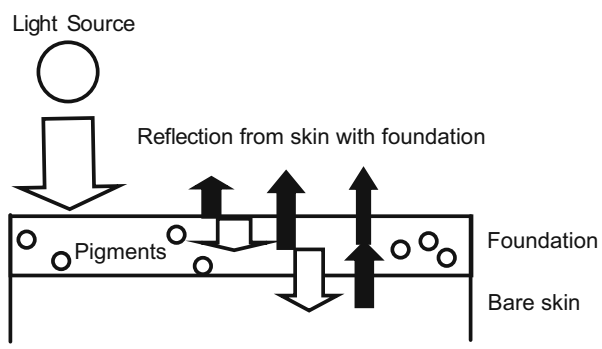

Fig. 2. Optics model for the skin with foundation make-up 


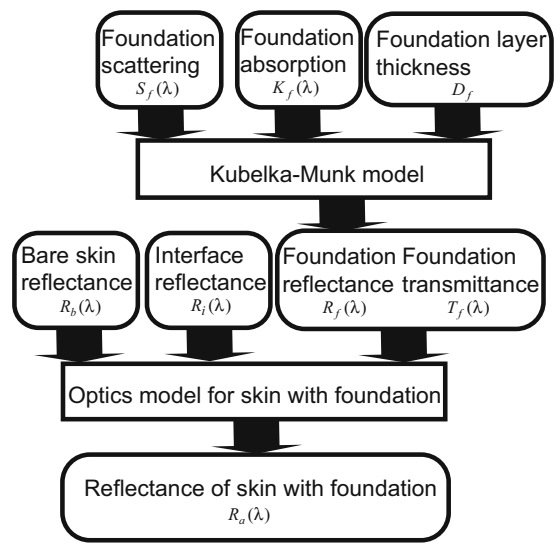

Fig. 3. Schema of spectral reflectance estimation for skin with foundation

where $S$ and $K$ are, respectively, coefficients of scattering and absorption in the media. When the object consists of two layers, multiple reflections in the interface between the higher layer and the lower layer is considered. In the twolayer model, the total reflectance including the inter-reflection is described as

$$
R_{1,2}=R_{1}+T_{1}^{2} R_{2}\left(1+R_{1} R_{2}+R_{1}^{2} R_{2}^{2}+\cdots\right)=R_{1}+\frac{T_{1}^{2} R_{2}}{1-R_{1} R_{2}}
$$

where $T_{1}$ and $R_{1}$ are the transmittance and reflectance of upper layer, respectively, and $R_{2}$ is the reflectance of lower layer.

The proposed algorithm can predict the spectral shape of skin surface by appropriately determining model parameters. Fig.3 shows the schema of the spectral reflectance estimation for skin with foundation make-up. We defined a set of equations for estimating spectral skin reflectance using the Kubelka-Munk theory as Equation 3.

$$
R_{a}(\lambda)=\left(1-R_{i}(\lambda)\right)\left(R_{f}(\lambda)+\frac{T_{f}^{2}(\lambda) R_{b}(\lambda)}{1-R_{f}(\lambda) R_{b}(\lambda)}\right)+R_{i}(\lambda)
$$

The functions of $\lambda$ in this equation are described as

$$
\left.\begin{array}{l}
R_{f}(\lambda)=\frac{1}{a_{f}(\lambda)+b_{f}(\lambda) \operatorname{coth} b_{f}(\lambda) S_{f}(\lambda) D_{f}} \\
T_{f}(\lambda)=\frac{b_{f}(\lambda)}{a_{f}(\lambda) \sinh b_{f}(\lambda) S_{f}(\lambda) D_{f}+b_{f}(\lambda) \cosh b_{f}(\lambda) S_{f}(\lambda) D_{f}} \\
a_{f}(\lambda)=\frac{S_{f}(\lambda)+K_{f}(\lambda)}{S_{f}(\lambda)}, b_{f}(\lambda)=\sqrt{a_{f}^{2}(\lambda)-1}
\end{array}\right\} .
$$

We note that the spectral reflectance $R_{a}(\lambda)$ is determined by the two parameters; the interface reflectance between the air and the skin surface $R_{i}$ and the thickness 
of foundation layer $D_{f}$. We assume that $R_{i}$ doesn't depend on wavelength. $R_{b}(\lambda)$ is the spectral reflectance of bare skin. $K_{f}(\lambda)$ is the absorption of foundation and $S_{f}(\lambda)$ is the scattering of foundation. It should be noted these coefficients depend on wavelength.

The CIE-XYZ tristimulus values are calculated using the estimated spectral reflectance of the skin, the spectral power-distribution of a light source, and the CIE color matching functions. These color values are further converted to RGB values by taking account of the characteristics of a calibrated display and the camera used for the bare face image.

\subsection{Division of Facial Image into Sub-images}

The input image of a bare face is divided into several sub-images. The size of the sub-image is $256 \times 256$ pixels. The image size is convenient for the MRA. The MRA process is done separately in eye areas, nose areas, a mouth area, forehead areas, and chin areas. Each sub-image includes overlap area to the neighboring sub-images. This area used for avoiding artifacts on edges in the texture control stage described later.

\subsection{Skin Color Detection and Conversion to Made-Up Color}

The detection of skin color and the conversion to made-up color are done for each sub-image. Terrillon [5] showed that the normalized r-g color space is effective for skin color-based image segmentation. The normalized $\mathrm{r}(n r)$ and normalized $\mathrm{g}$ $(n g)$ are defined as :

$$
n r=\frac{R}{R+G+B}, n g=\frac{G}{R+G+B}
$$

First, we extract skin area from the bare facial image. To perform this, all pixel values are mapped into the normalized $\mathrm{r}-\mathrm{g}$ color space, and the range of skin color is determined. Next, we estimate the make-up color to each pixel included in the extracted skin area. Note that all the pixel colors are determined on the basis of the make-up color estimated for a particular point on the cheek which is obtained from the surface-spectral reflectance analysis. Moreover, it is suggested that the whole of human face with make-up foundation has a reduced color distribution, compared with the original bare face.

Let $(R(x, y), G(x, y), B(x, y))^{T}$ be the original pixel values at location $(x, y)$ on the bare facial image, $\left(R_{c}, G_{c}, B_{c}\right)^{T}$ be the original pixel values at a particular point on the cheek, and $\left(R_{e}, G_{e}, B_{e}\right)^{T}$ be the made-up color values estimated from the spectral reflectance at the same point. Then the make-up color values at any location $(x, y)$ can be estimated in the following form.

$$
\left(\begin{array}{l}
R^{\prime}(x, y) \\
G^{\prime}(x, y) \\
B^{\prime}(x, y)
\end{array}\right)=\left(\left(\begin{array}{l}
R(x, y) \\
G(x, y) \\
B(x, y)
\end{array}\right)-\left(\begin{array}{l}
R_{c} \\
G_{c} \\
B_{c}
\end{array}\right)\right) \times \alpha+\left(\begin{array}{c}
R_{e} \\
G_{e} \\
B_{e}
\end{array}\right),
$$

where $\alpha$ is a weighting coefficient for adjusting color distribution. 


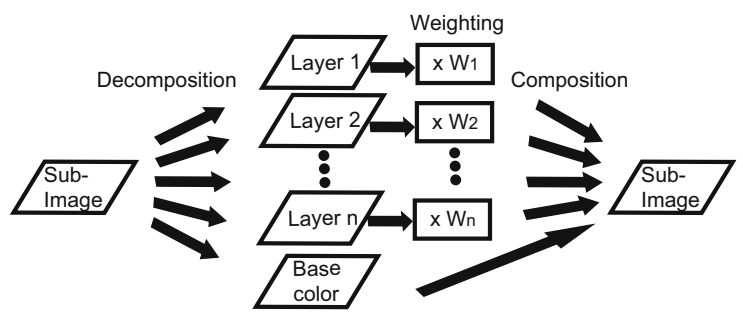

Fig. 4. Schema of texture control

\begin{tabular}{|c|c|}
\hline LL & $H L$ \\
\hline$L H$ & $H H$ \\
\hline
\end{tabular}

(a) Level -1

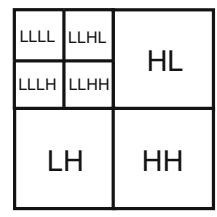

(b) Level -2

Fig. 5. Multi-resolution analysis

\subsection{Texture Control}

Fig 4 shows the procedure for texture control. Each sub-image with the estimated made-up color is decomposes into some texture layers by the MRA. Then, the texture image at each layer is weighted to change the intensity. Finally, these layers are combined into one sub-image.

Tsumura 6 proposed skin melanin texture control based on the Laplacian pyramid decomposition. In the present study, the wavelet decomposition with the Daubechies filter provides smooth synthesis of several textures on skin.

The wavelet transform is suitable for extracting both periodic patterns and local non-periodic patterns, that is, the feature patterns are localized in both spatial domain and frequency domain. Therefore, the MRA by wavelet transform is useful for extracting base color in the DC component and some feature patterns in color texture images. The MRA is done as the following steps. First, an image is decomposed into four components as shown in Fig. 5(a). These are the four components of low frequency for column and row (LL), low frequency for column and high frequency for row (LH), high frequency for column and low frequency for row (HL), and high frequency for column and row $(\mathrm{HH})$. The layer of Level-1 represents the composition of the original image into LH, HL, HH. This level includes the highest frequency component of the image. Second, the LL component is decomposed into the four components of Level-2 layer as shown in Fig. 5(b). Suppose a squared image of $2 \mathrm{~m}$-by- $2 \mathrm{~m}$ pixels. By repeating the decomposition, we obtain the multiple layers of Level-1 to Level-m with 2-by-2 and the DC component with 1-by-1. 


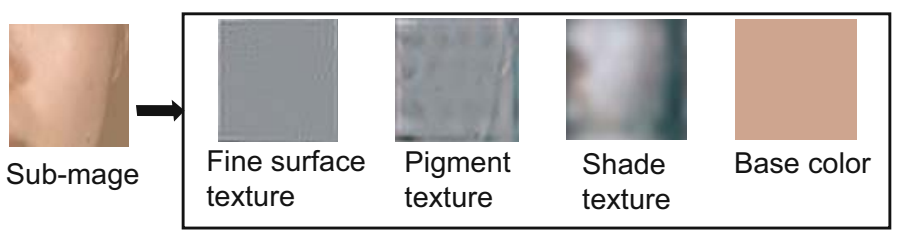

Fig. 6. Texture components of face image

The DC component shows the base color of the original image. The low frequency layers can include the shading of image. The middle and high frequency layers can include feature patterns of textures. Various texture patterns appear on the surface of human skin. For the whole made-up face image, we define four components. The components are (1) base color, (2) fine surface texture including pores, (3) pigment texture including acnes, and (4) shade texture caused by $3 \mathrm{D}$ face shape. Fig.6 shows the texture components. The color image of each texture component in the figure is enhanced for the explanation. In this case, we find that the fine surface texture exists in layers of Levels 1, 2 and 3. The pigment texture exists in layers of Levels 4 and 5 . Shade texture exists in layers of Levels 6,7 and 8 .

The decomposed layers are enhanced and attenuated with the weighting coefficient. The enhancement of the fine surface texture causes the enhancement of luster, and the attenuation of the pigment texture causes the attenuation of acnes. Then, the decomposed and texture-controlled layers are combined into one sub-image.

\subsection{Unification of Sub-images}

The texture controlled sub-images are unified into a whole face image. In this unification, the overlap areas are cut off. Finally, the skin area with the estimated made-up skin colors and the non-skin area in the original bare face image are combined into the whole face image as a synthesized made-up face image.

\section{$3 \quad$ Experiment}

Experiments were executed for evaluating the proposed method. First, we took two pictures of the bare face and the foundation-applied face for a person (Subject 1) by a digital camera. We analyzed the pictures and determined the texture control parameters. Next, we took the two kinds of picture for another person (Subject 2). Moreover, the surface-spectral reflectances of the bare cheek and the foundation-applied cheek were measured by a spectrophotometer. The made-up face image is synthesized from the bare face image and the spectral reflectance of the bare cheek. The image size of a facial image was 709x928 pixels . 
Table 1. Texture control parameters

\begin{tabular}{llll}
\hline Level & \multicolumn{3}{c}{ Weighting coefficient } \\
& R & G & B \\
\hline 1 & 3.00 & 3.00 & 3.00 \\
2 & 1.30 & 1.30 & 1.30 \\
3 & 1.00 & 1.00 & 1.00 \\
4 & 0.94 & 0.77 & 0.78 \\
5 & 0.82 & 0.70 & 0.65 \\
\hline
\end{tabular}

\subsection{Decision of Texture Control Parameters}

Sub-images of the facial image of Subject 1 were decomposed into multiresolution layers. The order of Daubechies wavelet was 8. Then, the standard deviations of the layers were calculated for each color components of RGB. The standard deviations of layers were compared between the bare face image and the made-up face image. The ratio of them was calculated. This ratio was used to determine the weighting coefficients in the texture control. Table 1 shows the weighting coefficients for Levels from 1 to 5 . Levels 1, 2 and 3 include the fine surface texture. The weighting coefficients for Levels 1, 2 enhance the luster. Level 4 and 5 include the pigment texture. The weighting coefficients for Levels 4 and 5 reduce the skin troubles, such as acnes. The coefficients for other levels were set to 1 . The color distribution control coefficient was set to 0.9 .

\subsection{Skin Color Estimation}

Fig. 7 shows the estimation result of the spectral reflectance for the made-up skin of Subject 2. The reflectance was estimated to fit the measured data with the parameter control on the interface reflection and the foundation thickness. Note

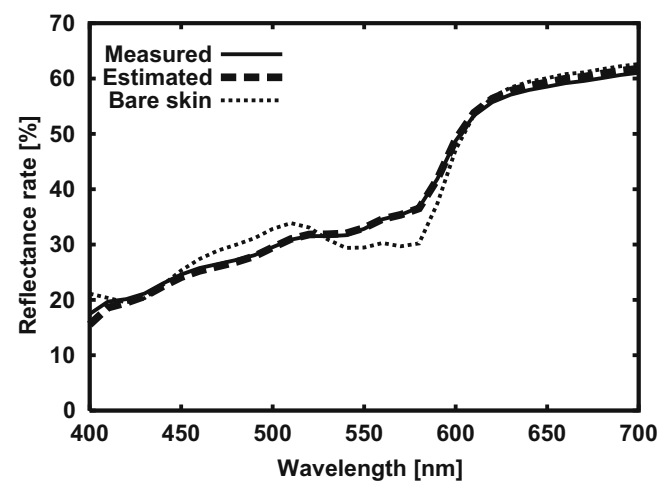

Fig. 7. Measured reflectance and the estimated reflectance of made-up skin 
that the spectral curve of the estimated reflectance is almost coincident with the measured curve. The average estimation error over the visible wavelength range was $0.5 \%$. Thus the accurate estimation of skin spectral reflectance is performed.

\subsection{Texture Control and Rendering Results}

The estimated spectral reflectances were converted to RGB values. Then, whole face image was synthesized by using the estimated colors and the texture control parameters shown in Table 1. In this case, the same control parameters were applied to all block images. Fig. 8 demonstrates a set of the bare face image, the

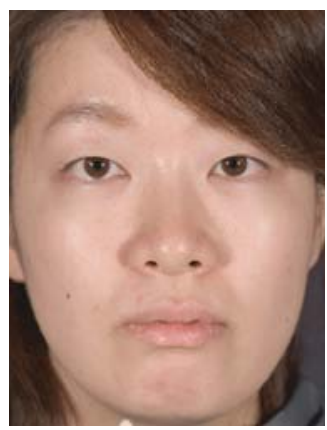

(a) Bare face

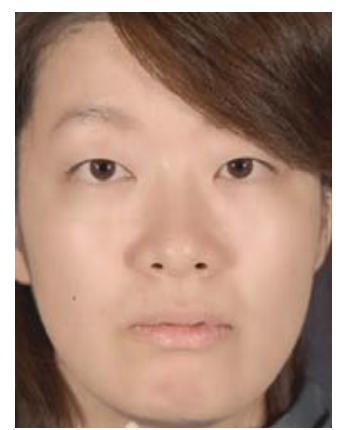

(b) Synthesized made-up face

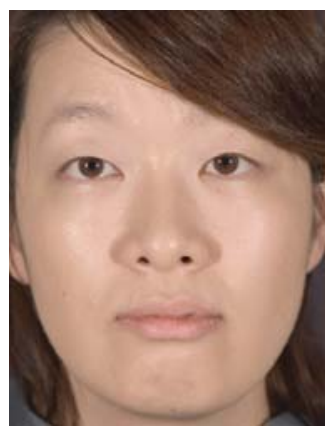

(c) Real made-up face

Fig. 8. Synthesized face image and real face images

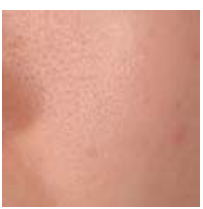

(a) Bare skin

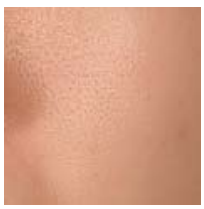

(b) Synthesized made-up skin

Fig. 9. Luster enhancement on cheek

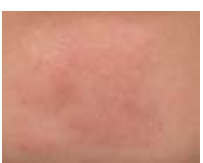

(a) Bare skin

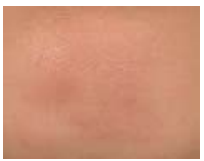

(b) Synthesized made-up skin

Fig. 10. Attenuation of acne patterns on chin 
synthesized made-up face image from the bare face image and the real made-up face image.

We should note that the synthesized made-up face image is very close to the real made-up face image. The proposed method predicts the made-up face image with sufficient accuracy. Figures 9 and 10 show the details in the images. Fig. 9 represents the luster effect on the cheek image, where the luster is enhanced at Layers 1 and 2. Fig. 10 represents the attenuation effect on the chin image, where reddish acne patterns in the synthesized image are inconspicuous. Specialists of cosmetics development gave the comment that the synthesized image showed the feeling of make-up on human face well.

\section{Conclusions}

This paper has described a method for synthesizing color images of the whole human face with foundation make-up by using bare face image and the surfacespectral reflectance of the bare cheek. The synthesis of made-up face images was based on the estimation of skin color with foundation make-up and the control of skin texture. First, the made-up skin color was estimated from the spectral reflectance of the bare cheek and the optical properties of foundation. The spectral reflectance of made-up skin was calculated by the Kubelka-Munk theory. Second, the texture control was based on the MRA with wavelet analysis. Smooth texture synthesis was done by the intensity change of layers in the MRA with the Daubechies wavelet. Luster was enhanced and acnes were attenuated by the texture control. Experimental results showed the accurate estimation results for made-up skin color and the effectiveness of the texture control. Thus, the proposed method predicts the made-up facial image with sufficient accuracy.

\section{References}

1. Doi, M., Tominaga, S.: Spectral estimation of made-up skin color under various conditions. In: SPIE/IS\&T Electronic Imaging, vol. 6062 (2006)

2. Doi, M., Tominaga, S.: Image Analysis and Synthesis of Skin Color Textures by Wavelet Transform. In: IEEE SSIAI, pp. 193-197 (2006)

3. Kubelka, P.: New Contributions to the Optics of Intensely Light-Scattering Materials. Part I. J. Opt. Soc. Am. 38(5), 448-457 (1948)

4. Kubelka, P.: New Contributions to the Optics of Intensely Light-Scattering Materials. Part II. J. Opt. Soc. Am. 44(4), 330-335 (1954)

5. Terrillon, J.C., Pilpre, A., Niwa, Y., Yamamoto, K.: Properties of Human Skin Color Observed for a Large Set of Chrominance Spaces and for Different Camera Systems. In: 8th Symposium on Sensing Via Imaging Information, pp. 457-462 (2002)

6. Tsumura, N., et al.: Real-time image-based control of skin melanin texture. In: ACM SIGGRAPH 2005 (2005) 